J. Sustain. Wireless Syst.

Vol.01/ No. 03

Pages: 198-205

http://irojournals.com/irosws/

DOI: https://doi.org/10.36548/jsws.2019.3.006

\title{
IMPROVISATION OF MESH NETWORK WITH WIDEBAND CODE DIVISION MULTIPLE ACCESS
}

\author{
Dr. D. Sivaganesan, \\ Professor, \\ Department of Computer Engineering, \\ PSG Institute of Technology and Applied Research, \\ Coimbatore, India. \\ Email: sivaganesan@psgitech.ac.in
}

\begin{abstract}
Mesh Networks are employed in various applications despite issues relevant to interference and network capacity. These networks also impose delay in routing and reconfiguration. Traditionally, TDMA and FDMA techniques have been used in these networks. This paper proposes wideband code division multiple access (WCDMA) technique for enabling maximum power transfer, low energy consumption, interference free and plugand-play feature enabling easy implementation of the technology. The spatial configuration and node count bounds are stated ensuring continuous satisfaction of quality of service by the communication link. Additionally, dynamic parallel orthogonal transmission and turbo coding schemes are used in combination to improve the efficiency of the network even for large hop counts.
\end{abstract}

Keywords: Mesh Network, WCDMA, wireless communication, energy efficiency, turbo coding

\section{INTRODUCTION}

Code Division Multiple Access (CDMA) is a mode of communication that uses multiple access technique. It allows simultaneous transmission of varied data through the same channel. A unique code containing information regarding the signal source and destination is assigned to each transition using speed spectrum. Wideband Code Division Multiple Access (WCDMA or W-CDMA), launched in Japan in 2001 is the communication standard that is frequently used in third generation $(3 \mathrm{G})$ cellular networks. It enables high-speed data transfer along with the basic MMS, text messaging and voice services. It facilitated mobile devices to access Internet broadband services.

Due to its high transmission rate, WCDMA is used for improving QoS. It can allocate multimedia traffic and handle services with large data rates. $144 \mathrm{kbps}$ to $2 \mathrm{Mbps}$ of data is supported by WCDMA technologies. Despite the primary use of WCDMA in mobile communication, the technology has found widespread application in the domain of wireless multiple access ranging from high-end computer networks to low-end wireless sensor networks. Communication networks called wireless mesh networks are composed of radio nodes that are organized in a mesh topology. The connectivity and speed of mesh networking can be enhanced by adopting WCDMA technology by interconnecting the users in the network without base stations. 
J. Sustain. Wireless Syst.

Vol.01/ No. 03

Pages: 198-205

http://irojournals.com/irosws/

DOI: https://doi.org/10.36548/jsws.2019.3.006

Using peer nodes, data packets are hopped to the destination in multi-hopping network. This helps reducing the inter-channel interference in mesh networking technologies used in wireless sensor networks. Modulation and demodulation of multiple packets can be done in parallel. Cautious power control algorithm are required for conservation of limited energy reserves in the nodes. In case of communication in areas with high density obstructions like high-rise buildings and trees, it is difficult to serve most of the customers due to line of sight (LOS) requirements. These drawbacks can be overcome by wireless mesh networks (WMN) as they provide wireless broadband internet facility.

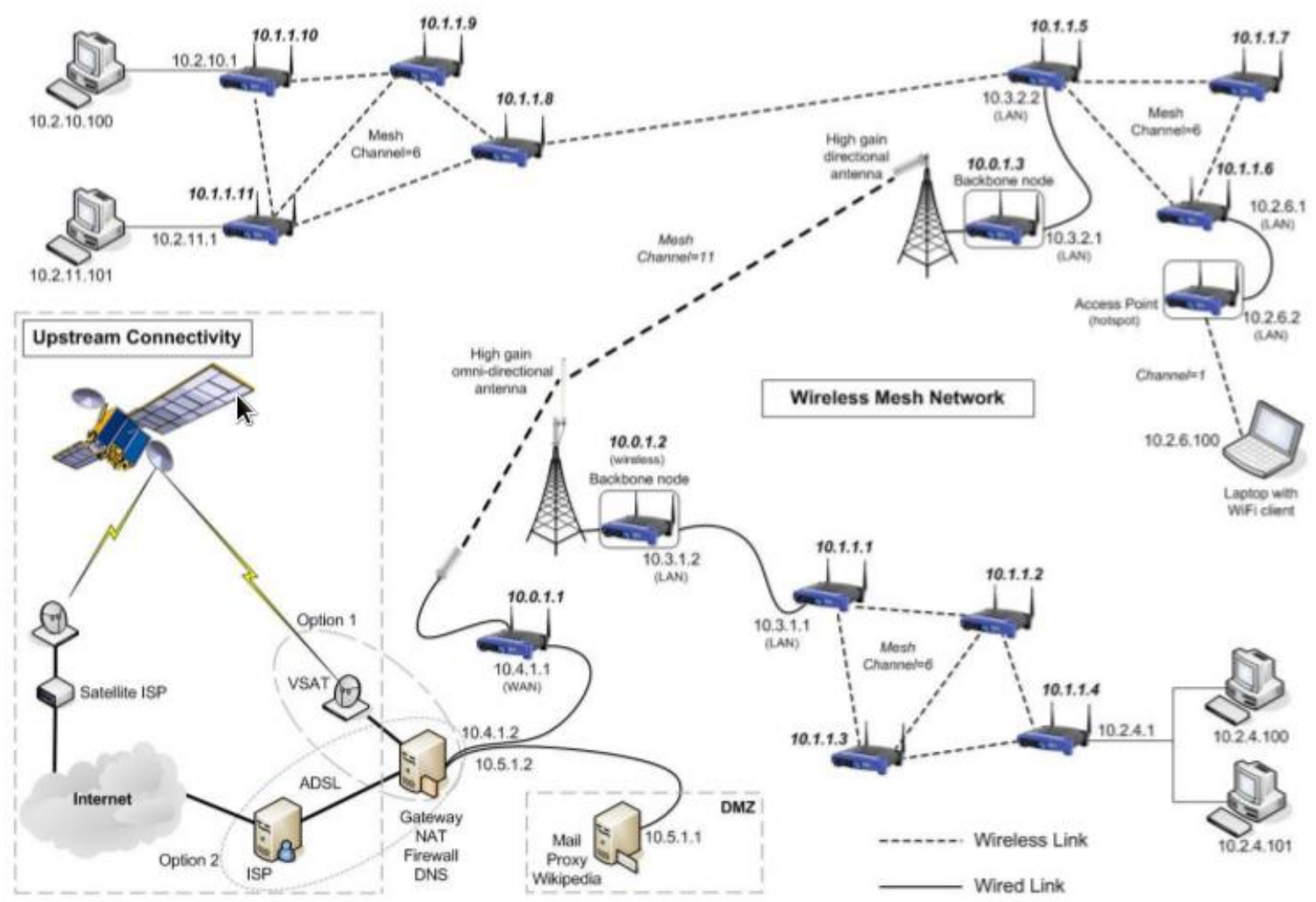

Figure 1: Wireless Mesh Network [22]

Figure 1 represents a wired-wireless mesh network configuration which has an upstream connection through a VSAT (Very Small Aperture Terminal) link. Details of the related work is provided in section 2. Section 3 provides information regarding the proposed work and Section 4 provides the simulation results. Finally, the conclusion and future work is briefed in section 5 .

\section{EXISTING LITERATURE}


J. Sustain. Wireless Syst.

Vol.01/ No. 03

Pages: 198-205

http://irojournals.com/irosws/

DOI: https://doi.org/10.36548/jsws.2019.3.006

Mihail et al. [21] presented the opportunities and challenges of wireless mesh networks and performed a study on the applications of WMN such as broadband internet access, indoor WLAN coverage, mobile user access and connectivity. This technology can successfully compete with traditional systems like WLAN, 3G, and WMAN. Woojoon Lee et al. [20] proposed a novel CDMA based multicast able switch and the hybrid NoC associated with it based on star and 2D-mesh topologies. This approach helped improve the efficiency of the network and to overcome the hotspot problem.

Leonardo Badia et al. [19] investigated optimization and management strategies for radio resources in WCDMA systems. The trade-off amongst price and utility is also considered. Utility functions are employed for accountability of added features characterized by traffic elasticity and further consider price effects in order to have accurate portrayal of economic quantities. Jagadeesh et al. [18] published a paper titled "Multiple Descriptions and Path Diversity for Voice Communications over Wireless Mesh Networks”. The authors perform the average distortion comparison in packetized communication per symbol using multiple description and path diversity for transmission of memory-less Gaussian source through additive white Gaussian noise channels. Further, the delivered voice quality is evaluated and compared with the existing methods.

Roman et al. [17] suggested the usage of wireless mesh networks in areas with infrastructure deficiency for providing internet access. The paper highlights the drawbacks of WCDMA networks that are based on 3G technology. Qualnet simulator is used for analysing the architectural capacity and reliability of the system. The macro diversity and interference between the base stations in WCDMA is discussed by Sana Ben et al. [14] for analysis of quality indicator matrices and their influence on the network design. The high data transfer rate of WCDMA improves the QoS in cellular networks [12]. The paper proposes improving signal strength with a power control scheme and connection admission control scheme for resource allocation.

Sihan Fan et al. [11] used orthogonal frequency division multiple access (OFDMA) for virtualization of resources in wireless mesh networks. Proportional fairness principle based physical resource allocation and load balancing based on virtual link multi-path mapping are the sub-problems of the system model. Mark Nixon et al. [10] studied the wireless mesh network that are used in real-time industrial field deployment. The WirelessHART mesh network that is used in research facility is explored in depth.

\section{PROPOSED WORK}

A wireless mesh network with $\mathrm{N}$ nodes that is inter connected is considered. Communication takes place between the nodes with the help of WCDMA spread-spectrum multiple access protocol. Here data is continuously broadcasted in place of node to node communication link maintenance. The mesh nodes consists of modulating 
J. Sustain. Wireless Syst.

Vol.01/ No. 03

Pages: 198-205

http://irojournals.com/irosws/

DOI: https://doi.org/10.36548/jsws.2019.3.006

and demodulating units in the form of transmitter and receivers. The architecture of the network, constraints in control, capacity of the network and the behaviour of the intended controller is described in this section. Figure 2 represents a simple architecture of multiple hop mesh network with multiple users.

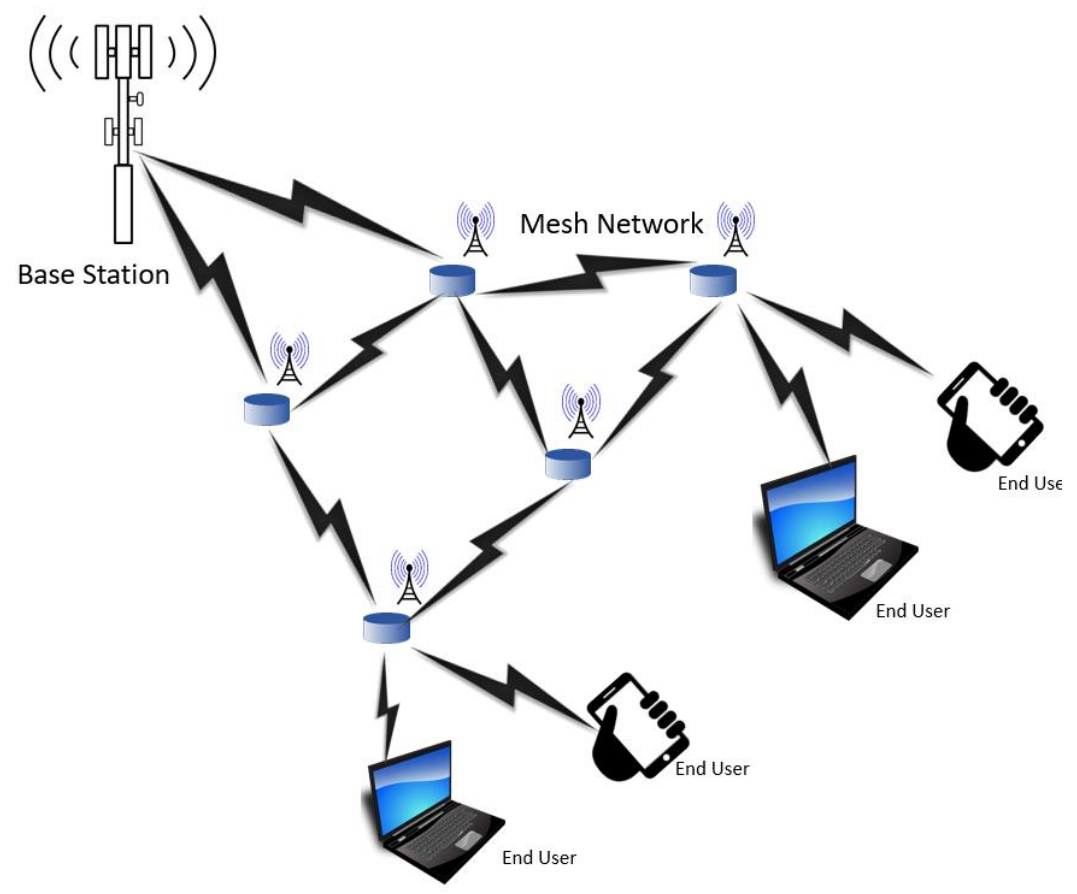

Figure 2: Multiple hop mesh network with multiple users

$$
\gamma_{i j}=\frac{P_{i} G_{i j}}{\sum_{k \neq i, k \neq j}^{N} P_{k} G_{k j}} \geq \gamma_{t}, \forall i, j, k \in \Omega
$$

Equation 1 represents the CIR constraint where $\mathrm{i}, \mathrm{j}$ and $\mathrm{k}$ are the nodes in the network. The transmission power levels of $\mathrm{i}^{\text {th }}$ and $\mathrm{k}^{\text {th }}$ nodes are $\mathrm{P}_{\mathrm{i}}$ and $\mathrm{P}_{\mathrm{k}}$, the link gains between the nodes are $\mathrm{G}_{\mathrm{ij}}$ and $\mathrm{G}_{\mathrm{kj}}$.

$$
P_{i} G_{i j} \geq R_{\text {min }}, \forall \mathrm{i}, \mathrm{j} \in \Omega
$$

Equation 2 represents the received power condition. In the above mesh network, the energy critical nodes perform power minimization using power control algorithm. This protocol can be very efficient for the end users since modulation and demodulation of multiple packets can be done in parallel. The signal to noise ratio of the system can be calculated with the following formula 
J. Sustain. Wireless Syst.

Vol.01/ No. 03

Pages: 198-205

http://irojournals.com/irosws/

DOI: https://doi.org/10.36548/jsws.2019.3.006

$$
\left(S N R_{n}\right)^{-1}=\left[\frac{1}{\frac{E_{b}}{N}}-\frac{n-1}{P G}\right]
$$

Where $\mathrm{n}$ is the number of spread packets, $\mathrm{PG}$ is the processing gain and $\mathrm{E}_{\mathrm{b}} / \mathrm{N}$ is the thermal noise. The delay jitter, average delay, bandwidth, bit rate and other major factors can be analysed.

\section{SIMULATION RESULTS}

With increasing node count, the behaviour of the Career-to-Interference Ratio (CIR) and the simulation results at every node is represented in Figure 3. The transmitted power across the node transmission power and node time step power.

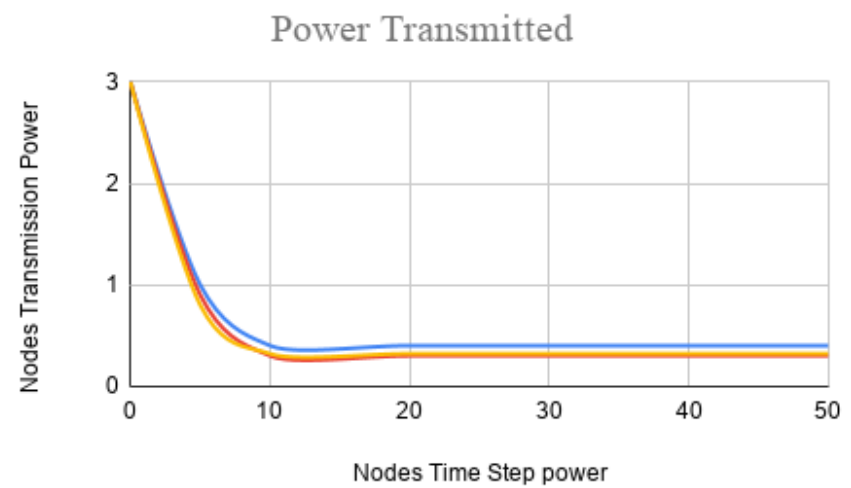

(a)

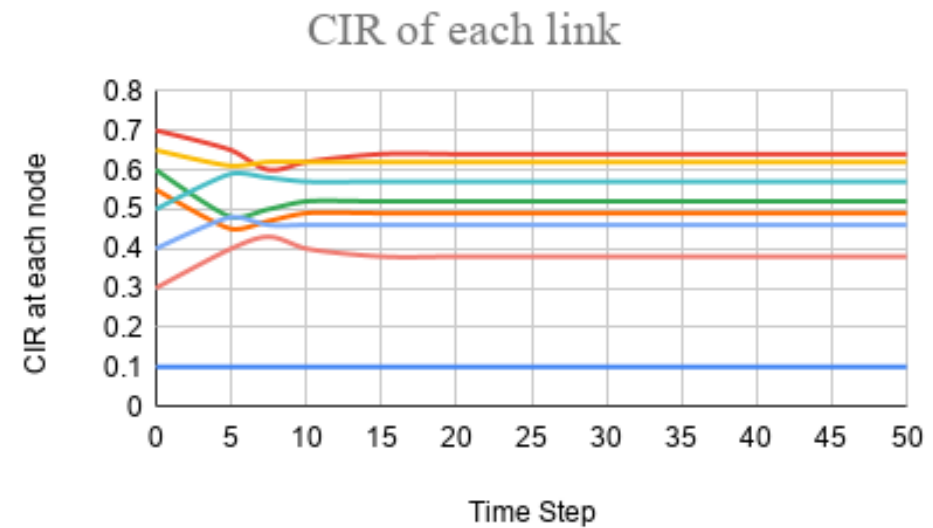

(b)

Figure 3: Transmission power control effects 
J. Sustain. Wireless Syst.

Vol.01/ No. 03

Pages: 198-205

http://irojournals.com/irosws/

DOI: https://doi.org/10.36548/jsws.2019.3.006

Form these graphs, it is evident that controller congregates to the minimum transmission power that satisfies all the restrictions defined in the previous section.

\section{CONCLUSION AND FUTURE SCOPE}

This paper proposes the implementation of wireless mesh networks with Wideband CDMA technique. A comparison with the traditional techniques like FDMA and TDMA is performed. The QoS criteria is also analysed for data and control packets as well as power saving aspects. This network is extremely suitable for consumer based applications. In case of use of narrowband CDMA, it can be used in defence and military applications. Low energy consumption, maximum power transfer, plug-and-play and interference free features has enabled easy implementation of this technology. Turbo coding and orthogonal transmission schemes are used for improved efficiency. Future work involves further reduction of power and improvement of efficiency. Application specific research is also proposed as future work.

\section{References}

[1] Andrews, Jeffrey G., Steven Weber, and Martin Haenggi. "Ad hoc networks: to spread or not to spread?[Ad hoc and sensor networks]." IEEE Communications Magazine 45, no. 12 (2007): 84-91.

[2] Akyildiz, Ian F., Xudong Wang, and Weilin Wang. "Wireless mesh networks: a survey." Computer networks 47, no. 4 (2005): 445-487.

[3] Patil, Rekha, and A. Damodaram. "A cross-layer based joint algorithm for power control and scheduling in CDMA wireless ad-hoc networks." WSEAS Transactions on Communications 8, no. 1 (2009): 122-131.

[4] Jiang, Hai, Ping Wang, Weihua Zhuang, and Xuemin Shen. "An interference aware distributed resource management scheme for CDMA-based wireless mesh backbone." IEEE Transactions on Wireless Communications 6, no. 12 (2007): 4558-4567.

[5] Prasad, Ramjee, and Tero Ojanpera. "An overview of CDMA evolution toward wideband CDMA." IEEE communications Surveys 1, no. 1 (1998): 2-29. 
J. Sustain. Wireless Syst.

Vol.01/ No. 03

Pages: 198-205

http://irojournals.com/irosws/

DOI: https://doi.org/10.36548/jsws.2019.3.006

[6] Andrews, Jeffrey G., Xiangying Yang, Aamir Hasan, and Gustavo de Veciana. "The flexibility of CDMA mesh networks." IEEE Wireless Communications Magazine (2005).

[7] Naveen, T. H., and G. Vasanth. "Qualitative study of existing research techniques on wireless mesh network." INTERNATIONAL JOURNAL OF ADVANCED COMPUTER SCIENCE AND APPLICATIONS 8, no. 3 (2017): 49-57.

[8] Daleel, Mubarak Elamin Elmubarak, and Marwa Eltigani Abubakar Ali. "Resources Management of Mobile Network IEEE 802.16 e WiMAX." INTERNATIONAL JOURNAL OF ADVANCED COMPUTER SCIENCE AND APPLICATIONS 8, no. 4 (2017): 262-269.

[9] Kashif, Muhammad, M. J. Hossain, Yuba Raj Kafle, Edstan Fernandez, SM Nawazish Ali, and Vivek Sharma. "Communication Architecture, Technologies, and Requirement for Modern Energy Systems." In 2018 Australasian Universities Power Engineering Conference (AUPEC), pp. 1-6. IEEE, 2018.

[10] Nixon, Mark, Paul Muston, Shaobo Zheng, Tao Gong, Eric Rotvold, Wally Pratt, and Song Han. "An empirical study of industrial real-time wireless mesh network in field deployments." In 2016 IEEE 14th International Conference on Industrial Informatics (INDIN), pp. 612-617. IEEE, 2016.

[11] Fan, Sihan, Xiaofeng Lu, Bowen Liu, Huan Liu, and Danping Zhao. "Resource Virtualization in OFDMA Wireless Mesh Network for Emergency Communications." In 2019 IEEE International Conference on Communications Workshops (ICC Workshops), pp. 1-6. IEEE, 2019.

[12] Khare, Vandana, Y. Madhavee Latha, and D. Sreenivasa Rao. "Adaptive Power Control for Quality of Service Improvement in WCDMA Wireless Networks." International Journal of Applied Engineering Research 12, no. 8 (2017): 1779-1783.

[13] Wang, Jingjing, and Chunxiao Jiang. "Machine Learning Paradigms in Wireless Network Association." Encyclopedia of Wireless Networks (2018): 1-9.

[14] Jamaa, Sana Ben, Hervé Dubreil, Zwi Altman, and Arturo Ortega. "Quality indicator matrices and their contribution to WCDMA network design." IEEE transactions on vehicular technology 54, no. 3 (2005): 11141121. 
J. Sustain. Wireless Syst.

Vol.01/ No. 03

Pages: 198-205

http://irojournals.com/irosws/

DOI: https://doi.org/10.36548/jsws.2019.3.006

[15] He, Jianhua, Kun Yang, Ken Guild, and Hsiao-Hwa Chen. "Application of IEEE 802.16 mesh networks as the backhaul of multihop cellular networks." IEEE Communications Magazine 45, no. 9 (2007): 82-90.

[16] Allen, Stuart M., Roger M. Whitaker, and Steve Hurley. "Personalised subscription pricing for optimised wireless mesh network deployment." Computer Networks 52, no. 11 (2008): 2172-2188.

[17] Seibel, Roman, Nils-Hendrik Klann, and Tim Waage. "Wireless Mesh Networks for Infrastructure Deficient Areas."

[18] Balam, Jagadeesh, and Jerry D. Gibson. "Multiple descriptions and path diversity for voice communications over wireless mesh networks." IEEE transactions on multimedia 9, no. 5 (2007): 1073-1088.

[19] Badia, Leonardo, Cristiano Saturni, Lorenzo Brunetta, and Michele Zorzi. "An optimization framework for radio resource management based utility vs. price tradeoff in WCDMA systems." In Third International Symposium on Modeling and Optimization in Mobile, Ad Hoc, and Wireless Networks (WiOpt'05), pp. 404-412. IEEE, 2005.

[20] Lee, Woojoon, and Gerald E. Sobelman. "Mesh-star hybrid NoC architecture with CDMA switch." In 2009 IEEE International Symposium on Circuits and Systems, pp. 1349-1352. IEEE, 2009.

[21] Sichitiu, Mihail L. "Wireless mesh networks: opportunities and challenges." In Proceedings of World Wireless Congress, vol. 2, p. 21. 2005.

[22] Johnson, David, K. Matthee, D. Sokoya, L. Mboweni, A. Makan, and H. Kotze. "Building a Rural Wireless Mesh Network: A do-it-yourself guide to planning and building a Freifunk based mesh network." Meraka Institutue (2007). 